\title{
The Study for Adapting Knowledge Sharing Technologies into Indian Education Market
}

\author{
Sneh Sagar and Song-Kyoo Kim, Senior Member, IACSIT
}

\begin{abstract}
The study is designed for the startup company to make their strategic decisions about expanding their business in Private Higher Education Institutions sectors. There are technologies present in India that could be used to conduct online educations. Current technologies and methods are far from being leveraged due to the orthodox mindset of teachers, parents and students. The research is targeted to demonstrate for showing how to adapt the new technologies for knowledge sharing into the practical level based on the real company cases and provides the guidelines for adapting knowledge sharing technologies more effectively.
\end{abstract}

Index Terms - Technology adaptation strategy, knowledge sharing, India, education.

\section{INTRODUCTION}

Education is an important means of modernization. The importance of education can be realized from the fact that all modernized societies tend to emphasize on universalization of education as a mode for sustained economic development. There has been a huge demand for upgrade of education as India expected to have a surplus of 47 million people in the working age group by 2020. As a result, both the formal education sector (including K-12 and higher education) as well as private sectors (including coaching institutions, pre-schools and vocational institutions) are witnessing rapid growth in India. The Indian education system, considered as one of the largest in the world, is divided into two major segments of core and non-core businesses. Private education sectors are the major market in non-core business area.

India Ratings have expected the Indian education sector's market size to increase to 602,410 INR (111.16 billion USD) by 2015 due to the expected strong demand for quality education. The sector had grown at a compounded annual growth rate of 16.5 percent during 2005-2011. The private education sector is estimated to reach 70 billion USD by 2013 and 115 billion USD by 2018. The higher education sector in India is expected to witness a growth of 18.0 percent CAGR untill 2020. Currently, the sector witnessed spends of more than 46,200 INR ( 8.52 billion USD), which is estimated to grow at an average rate of more than 18.0 percent to over 232,500 INR (42.89 billion USD) in 10 years [1]. The Indian education system has been dominant with the face-to-face teaching methodology though globally there was a growing trend of the online classes [2]. In the United States alone approximately 31 percent of the college students used online

Manuscript received March 12, 2014; revised June 12, 2014

The authors are with Asian Institute of Management, Makati City, Philippines (the corresponding author: Song-Kyoo Kim; tel.: +632-892-4011; e-mail: SKim@aim.edu). classes [3]. Specially, the market of the Private Higher Education Institutions (PHEI) in India [4] are growing tremendously (see Fig. 1).

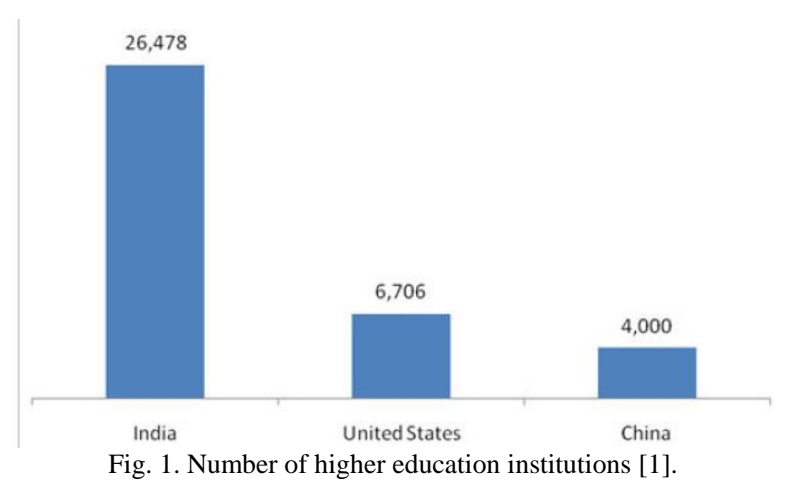

Face-to-face teaching method in PHEI sectors of India has been being followed since time immemorial and the online or 'virtual' classes were sparsely present. In fact, the online method of education for students is far from being envisaged. People in India felt that face-to-face classes offer a social experience, personal connections, structured learning and consistent communication which are critical for overall academic development of a student. They feel that personal attention given to a student by the teacher helps the student understand anything better. Even though, the teaching method is transformed into online educations. Face-to-Face based teaching is still dominant method in India because of their social cultures. As the startup company in India, they can have the following options for expanding their business: 1) Similar approach of other companies, involves hiring new faculties for various cities. The two existing faculties will train the new hires because the institute has its own unique teaching methodologies. After receiving the training the new faculties will shift to new cities to conduct the classes. 2) Building up e-Learning (online education) system to telecast the classes from the base city i.e. Bangalore to different cities in the country. It is the part of technology adaptation for knowledge sharing within various cities that is the actual choice from one of the startup company.

\section{MARKet COMPETITIONS}

In PHEI (Private Higher Education Institutions) of India, there are continuous tussle in which each player has tried to acquire students from other players. Success in this industry is hugely dependent on national reach. The more a company has branches the more number of students it is able to cater. Due to the presence of other players at the same location, every company tries to bring down the price it charged to the students. This leads the companies to offer discounts in order 
to attract students. Newspaper commercials have been the major medium through which all the companies have done their marketing. Huge banners and posters are also a part of the marketing strategy. As this market become more and more mature, the companies understand that technology and innovation could be the part of capturing the market share. Again, there are a huge market which composed of students who are not aspiring for an MBA degree. Companies capture this genre it has been critical to come up with programs that would help students preparing for other entrance exams (Fig. 2).

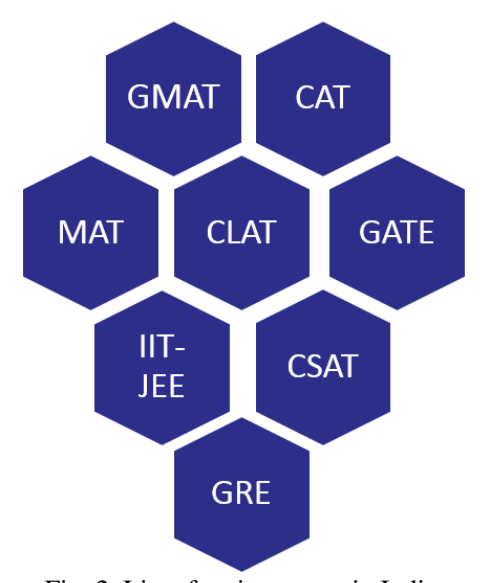

Fig. 2. List of major exams in India.

\section{A. Career Launcher}

Career Launcher (CL) has been Asia's leading education service provider led by a team of IIT-IIM alumni, with a passion for education. Since its inception in 1995, it had focused on academic excellence and domain expertise to work towards its core objective of enabling individuals to realize their potential and making their career dreams come true. Over the last 15 years, CL had established itself as a national player in the field of test preparation. It functioned across a broad spectrum of educational services including K-12 schools, higher education and vocational training. CL was present in $75+$ cities/towns of India with educational infrastructure in the form of learning centers in excess of 160 in number. The total inclusive manpower of CL was in the region of about 3000 with $65 \%$ of this being only teachers/trainers/academicians. CL also had international presence in Dubai and Abu Dhabi and offered various educational services to its students in these countries. CL was very price-competitive and offered packages which were very attractive to the students. A typical package included classes, study material, test series, mock exams and mock interview sessions.

Smart CAT Cracker is a tailor-made product for students who wanted to imbibe the ins and outs of aptitude testing and become adept at applying shortcuts and using options to arrive at answers quickly. The module taught methods of attempting questions while at the same time giving the student a rerun through key concepts. In addition to the conventional methods, students can learn how to crack questions with aptitude that helps a student to improve his speed and, consequently, his performance across various management entrance exams such as CAT, XAT, IIFT, CMAT, SNAP, NMAT and others (Fig. 2). Smart CAT
Cracker consists of 240 sessions, divided into 5 modules, which captured the teachings of prominent experts of the field of aptitude testing. The meticulously selects questions in these modules give a flavor of actual exam questions. It is essential to go through each of the questions in the post-exercises diligently so that the concepts they need.

\section{B. Triumphant Institute of Management Education}

Triumphant Institute of Management Education Pvt. Ltd. (TIME) is one of leading test-prep institute with a pan-India presence and is headquartered at Hyderabad. Established in 1992, TIME has operated out of 216 offices located in 110 towns and cities across the country. Over 40 IIT/IIM graduates formed part of the core team at TIME. TIME is acknowledged as a multi-location, multi-program training specialist run on corporate lines and offers a wide range of programs not only for national and state-level entrance exams like the CAT, MAT and IIT-JEE, but also for international exams like GMAT, GRE and TOEFL. The price at which TIME offers its courses was almost equal to that of Career Launcher. Through its Campus Recruitment Training conducted at various engineering colleges across the country every year, TIME trains thousands of students in aptitude tests and communication skills as a part of preparing them for their campus placements. In keeping with its tradition of always maintaining a watchful eye on market trends and tapping them, TIME has introduced training programs for GATE, CSAT and CLAT. More than 75,000 students have trusted TIME for their preparation for the CAT 2011 exam. Marketing has been critical in increasing the market share of an institute and thus TIME has used to spend a considerable amount in marketing through its newspaper and TV commercials.

\section{IMS}

IMS is another leader in management entrance training. IMS has spread its wings across India with 120 centers and over 50,000 students, successfully making it to some of the most prestigious institutes in India. The institute charges a premium price from students because of the quality of teachers it employed.

Mr. Rane who was the founder of IMS came to Mumbai in 1960s and was appointed as a Lecturer of English in HR College of Commerce and Economics where he taught for about 7 years. In 1970, IIM Ahmedabad appointed him as a part-time lecturer in the Written Analysis and Communication department for their Business Writing course. For 2 years, he traveled between Ahmedabad and Mumbai. During his journey, he had seen young students confused and clueless about career avenues that a small town education offered. All over India, there were those who had the resources for an international education but were not aware about courses available, entrance exams and other requirements and whom to approach for help with all of this. He realized that in India, a huge talent pool was getting wasted. In the late $70 \mathrm{~s}$, he released the first set of material of its kind to enable attempting the CAT and later went on to add GRE, GMAT and TOEFL, which he painstakingly prepared himself. He was not only identified MBA as an educational opportunity well before the current craze, but also identified key success factors in a business, which dealt 
with young, highly intelligent minds.

Currently, IMS charges students with premium fees as in most cases it conducts its classes at prime locations of the city. For marketing, it uses to take the support of newspaper commercials but marketing expenses are not as much as TIME or Career Launcher.

\section{Educomp}

Educomp is one of the largest education companies in India and the only company spread across the entire education ecosystem. Its presence in each of the education verticals is marked with technology-enabled products, high quality people, structured processes and entrepreneurial leadership, that came together to deliver unmatched value. Founded in 1994, Educomp has been a leader in digital content solutions for the K12 segment.

The smartclass Class Transformation System (or CTS) and the smartclass Digital Teaching System (or DTS) are the biggest and most innovative initiatives in the space of digital classroom content and digital classroom hardware respectively. The company has entered US and Asia Pacific markets through acquisitions and partnerships. Their subsidiary, Wizlearn Technologies (formerly known as ASKnLearn) focused on Pan Asian market place is based out of Singapore. Wizlearn also catered to institutions of higher learning and corporate clients in Singapore, China, Thailand, Philippines, Indonesia and Brunei. Their subsidiary Learning.com is the leading web based K12 Company in USA. In Middle East our Joint Venture Company is called Educomp Arabia Co Limited. The backbone of their expansion across the global value chain was a strong $R \& D$ dedicated to creating quality contents and IP to serve the entire education ecosystem. Smartclass online is a portal for students studying all over the world. A student has exchanged and shared your notes, learning materials, tricks and tips with other students. They could make friends, and communicate with them via Smartclass section that also be a virtual for Educomp smartclass schools which provide instructor-led education solutions for private schools, and has been developed as one of largest content library and classroom session in India.

\section{E. Think and Learn}

Think \& Learn Pvt. Ltd is the startup company who has been recently entering PHEI sectors in India. This company, earlier known as Noesis Education and Management Services is the parent company of various nationally recognized brands such as Byju's CAT Class, Byju Civil Services Aptitude Test (CSAT) Class, and Entrance Toppers and so on. The company has been a market leader in Bangalore for CAT preparation. It was founded by Byju Raveendran in 2007 to change the current paradigm of competitive exam training. Byju Raveendran was the two time 100 percentile in CAT and a national Math Olympiad winner. At the young age of 26 , he trod the path less travelled by giving up a prestigious seat in the IIMs and a lucrative corporate career to change the way people Think \& Learn. Under his visionary leadership along with his unending passion for teaching, Think \& Learn had grown from its modest root of just 100 students in 2007 to a national level brand. He was known for his unconventional teaching techniques and rose to be the foremost trainer in the competitive exam training in the entire country. His vision and purpose gave the genesis of the several famous brands which has revolutionized the way learning and teaching is imparted. Vastly experienced and qualified faculty pool of the company took care of the various courses offered a hugely differentiated learning experience which inspired the students to take an exhilarating learning ride with highest involvement resulting in higher ratio of success. The success record in various competitive exams became the new standards and certifies the efficacy of the teaching methodology. Its mission through test preparation is not only to ensure higher success ratio in maximum conversion but in process culminate a sense of learning and self-awareness through its motto of unconventional route to teaching.

According to the current market research for PHEI market, Career Launch and TIME are the main players in the industry and move onto online education sector very quickly (see Fig. $3)$.

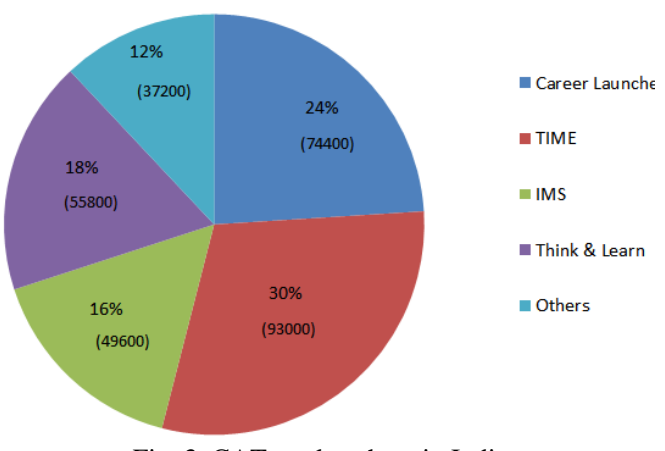

Fig. 3. CAT market share in India.

Fig. 4 and Fig. 5 are the competitor analysis results that show the positioning of the competitors on the strategic maps. The size of the circle corresponding to each company in the maps shows the market size of the company. Product diversity indicates the number of courses offered by each competitor. It is a crucial factor in any growth of the company because if the number of courses offered are more, more students would enroll for them and the company is able to increase their market presence. National reach indicates the number of centers each company has in India and price indicates the average fee charged by the companies for various courses.

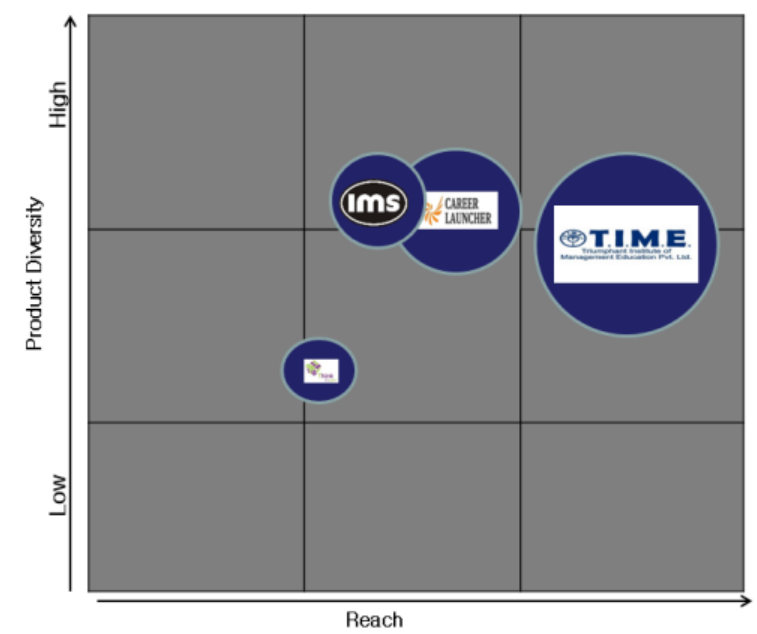

Fig. 4. Strategy map I. 


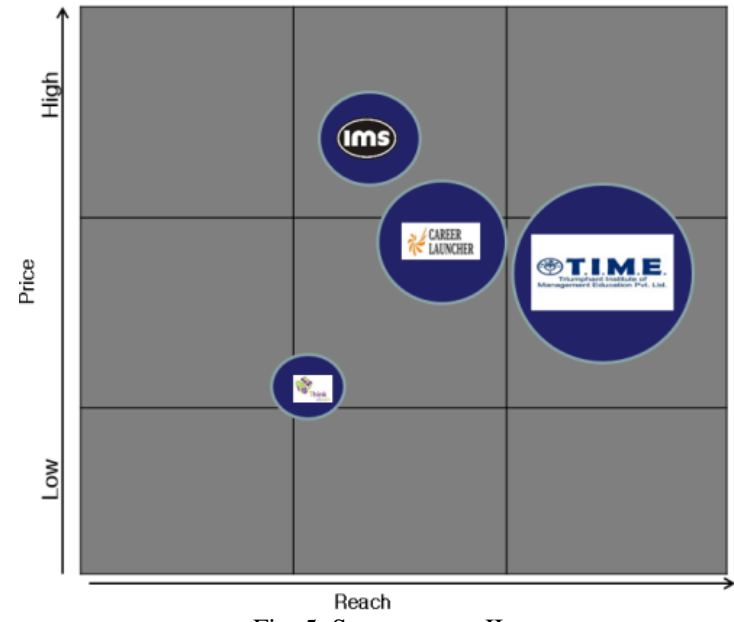

Fig. 5. Strategy map II.

\section{TECHNOLOGIES FOR ONLINE EDUCATIONS}

The term of e-education (or online education) refers to the use of Internet technology to deliver classes to students at a remote location. Online courses are ideal for students whose work schedule or family commitments do not allow them to attend classes on campus. In order to expand learn online educations, students require self-discipline and their own motivation. There is an increasing awareness for online educations even though offline system has been dominant in India. The infusion of ICTs (Information Communication Technologies) into learning and teaching have occurred in all sectors of education. It has changed the nature of face-to-face teaching and enabled the rapid growth of blended and online courses. ICTs offer new opportunities but also new challenges for both instructors and students. Technologies enabled instructors, students and others to participate in teaching and learning at a time and place convenient to them. SWOT (Strengths, Weaknesses, Opportunities, and Threats) analysis of online education provides the strategic analysis of adapting the technology in details (see Fig. 6).

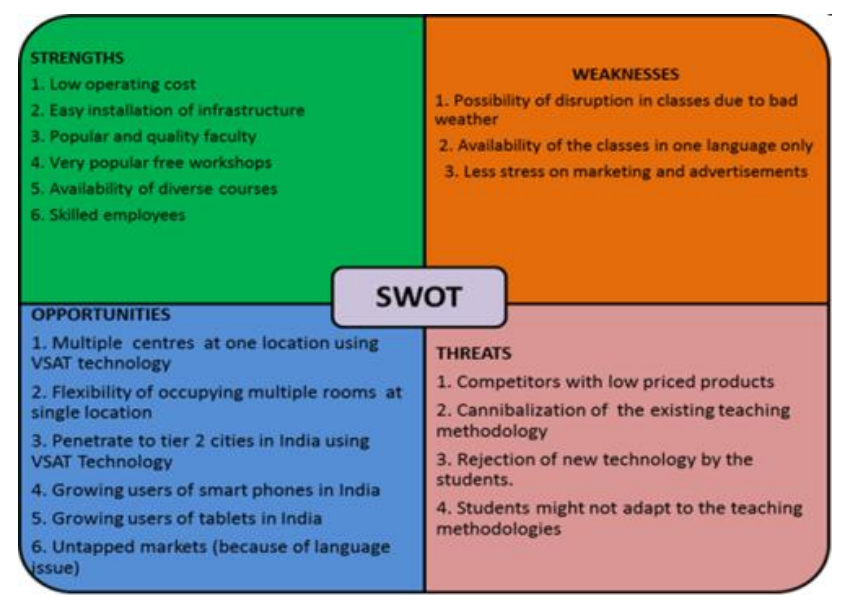

Fig. 6. SWOT analysis of online educations.

The on-demand streaming method is usually video based material which is similar to a face-to-face classroom course, with recorded lecturer speaking and PowerPoint slides. Video streaming technologies are applied and students can watch the video by means of freeware or plug-ins (e.g., Windows Media Player or RealPlayer). Animations can be also applied which is a good way of making the content and its appearance more interesting which is most widely used means of online education.

Live streaming may be the alternative based on the video streaming technologies. When a lecture is delivered at the source site as in a distance education application, it is encoded on the fly using hardware based encoding solution for sending to the clients. In this case, the source site typically hosts the encoding hardware component. The encoded file is sent to the streaming server placed at the Internet Data Centre (IDC) of Internet service providers for serving the clients as real time basis. During live encoding, the file is also stored for future use. The encoder (typically hardware based) compresses the audio/video source in real time and sends it to the streaming server. The live compression to various bit rates could also be done simultaneously depending on the encoder being used. In case the encoder is a part of the streaming server itself, it directly starts streaming the compressed file. The live streaming could also be stored on the server and could be used for streaming in the on-demand mode in the future.

\section{TECHNOLOGY ADAPTATIONS FOR ON-DEMAND STREAMING}

Recorded contents can be encoded off-line at different rates and uploaded to the server. Valid users after authentication can start the streaming session at any time convenient. As the authorized users, they have the options to pause, rewind, fast forward and so on. In the case of live streaming, as the contents are played in the real time from the source site, additional care required to ensure acceptable Quality of Service (QoS) in terms of delay, loss and delay jitter parameters. On-demand streaming provides flexibility to users to access the content from their desktops at any time. In both these cases, once the streaming session starts, users expect continuous and jitter-less transmission. Assuming source material recorded on CDs, this contents could be compressed and encoded in various bit rates (56 kbps, 150 kbps, $256 \mathrm{kbps}$ ) and formats (*.mp4, *.mov), which suitable for streaming (Fig. 7). Registered participants are given the URL to view the lecture after authentication. Participants access the streaming site and select the appropriate bit rate link based on their network connectivity before watching lectures. Standard players which supported streaming such as QuickTime, Real, Windows are only required for watching lectures.

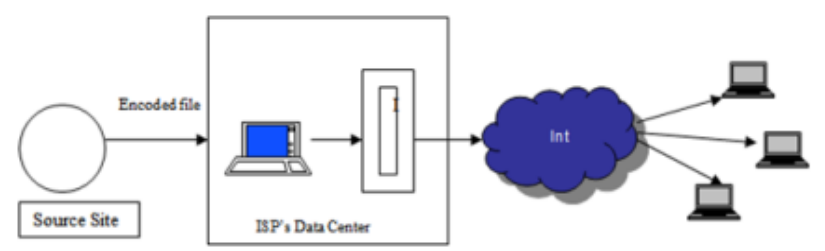

Fig. 7. Networks for online educations.

Unlike Live Web Streaming, On-demand Webcast is a pre-recorded presentation, which is available on-line at the viewer's convenience. The video is edited and can be mixed with presentation slides. Alternatively, we can synchronize the slides side by side next to the video, creating a fully featured online presentation. Both of technologies have their 
own benefits (see Table I).

TABLE I: INTERNET VIDEO TECHNOLOGIES

\begin{tabular}{|l|l|}
\hline \multicolumn{1}{|c|}{ Live Web Streaming } & \multicolumn{1}{c|}{ On-Demand Webcasts } \\
\hline $\begin{array}{l}\text { 1) Time-critical content can be } \\
\text { broadcasted to a global audience. }\end{array}$ & $\begin{array}{l}\text { 1) It offers global reach and different } \\
\text { time zones are not an issue. }\end{array}$ \\
$\begin{array}{l}\text { 2) Audience is more engaged as if they } \\
\text { were present for the actual lecture. }\end{array}$ & $\begin{array}{l}\text { 2) The viewer can watch the presentation } \\
\text { wherever they wish to. }\end{array}$ \\
$\begin{array}{l}\text { 3) Global viewers can engage with the } \\
\text { presenters through live online Q\&A. }\end{array}$ & $\begin{array}{l}\text { 3) The footage can be edited and cleaned } \\
\text { up before going on-line. }\end{array}$ \\
& $\begin{array}{l}\text { 4) The presentation can be paused, } \\
\text { rewound and repeated. }\end{array}$ \\
& $\begin{array}{l}\text { 5) A link to the webcast can be sent to } \\
\text { your contacts by email or social media. }\end{array}$ \\
\hline
\end{tabular}

\section{Very Small Aperture Terminal (VSAT) TECHNOLOGY}

A very small aperture terminal (VSAT), is a two-way satellite ground station or a stabilized maritime VSAT antenna with a dish antenna that is smaller than 3 meters [5]. The majority of VSAT antennas range is between 0.75 meters and 1.2 meters. Data rates typically range is from $56 \mathrm{kbit} / \mathrm{s}$ up to $4 \mathrm{Mbit} / \mathrm{s}$. VSAT satellite(s) in geosynchronous orbits to relay data from small remote earth stations to other terminals (in mesh topology) or master earth hub station (in star topology). The VSAT communication technology has emerged to eliminate the digital divide between nations and significantly improve the quality of life by enabling worldwide access to global information resources. The VSATs are a compact satellite dish mated with the necessary electronic hardware to create an earth station. The earth station consisted of a small antenna and other devices to interface between the antenna and the end-user equipment. The end-user equipment could be one or more personal computers (PCs), telephone, a PBX, a video receiver/player or any combination of these. VSAT could receive or in another configuration, receive and transmit signals. A VSAT could link a few or if necessary, hundreds of sites, including geographically remote locations (Fig. 8).

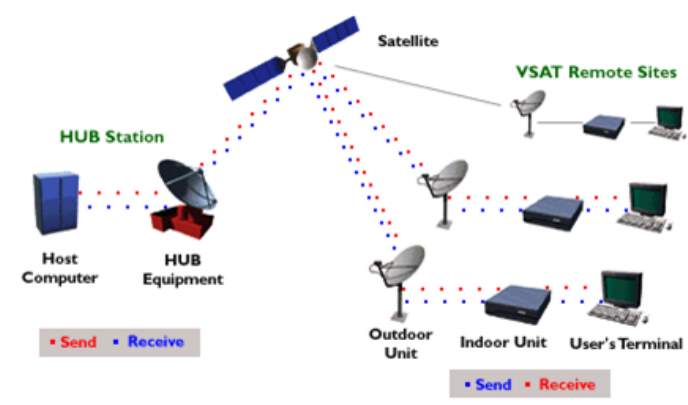

Fig. 8. VSAT based network architecture [6].

A VSAT supports a variety of network configurations and large information including computer data that could be transmitted at different speeds [7]. The VSAT is more flexible and less expensive than many land-based communication networks and it is useful for institutions/universities with multi campuses. Due to its dependency on wireless communication transmission medium, VSAT has been overcome many problems of telecommunication. It is a viable technology when terrestrial lines were costly and difficult to install. As a result, there is no need to invest in high capacity transmission lines, which are very difficult to create to cover the entire country. VSAT provides cost effective, reliable telecommunication over large geographical areas, replacing or supplementing traditional terrestrial lines. Telephone and services such as e-mail and data transfer via VSAT did not require the use of radio frequencies. Because of VSAT technology adaptation, on-demand streaming become very attractive to existing terrestrial networks in terms of availability, performance, variety of services, cost per byte transmitted and cost pet incremental capacity growth.

\section{CONCLUSION}

The major target of this project is demonstration of the strategic decision making for the knowledge sharing technology adaptation in Indian education market. It also shows the insights of education markets in India with their major key players in private education sectors. The market analysis of India such as SWOT analysis and competitive analysis are included in this research to help for understanding the insights of the private education sectors in India.

\section{ACKNOWLEDGMENT}

This paper is based on the case research of Asian Institute of Management, titled "Think and Learn Pvt. (A) (B)" which are originally designed for the case room discussion of strategy and technology management courses for MBA students.

\section{REFERENCES}

[1] M. M. Raju et al., Annual Report 2009-10, Ministry of Human Resource Development, Government of India, 2010.

[2] Ernst and Young, "40 million by 2020: Preparing for a new paradigm in Indian Higher Education," Ernst and Young - Edge 2011 Report.

[3] Going the Distance: Online Education in the United States, 2011.

[4] V. Yallapragada and S. Vutukuru, "Private higher education institutions (phei) and necessity of e-governance," International Journal of Management, IT and Engineering, vol. 2, no. 9, 2012, pp. 192-198.

[5] G. Heifner, "Introduction to VSAT Technology, Broadband Properties," March 2004, pp. 24-29.

[6] Telstream Telecoms. What is VSAT, Telstream Telecoms Website? [Online]. Available: http://www.telstream.co.za/support-2/what-is-vsat/

[7] Afri Connect, SatIP4 Low Cost C / Ku Band IP VSAT Solutions, Company Brochure, 2011, p. 2.

Sneh Sagar was a member of Innovation and Operations Laboratory in Asian Institute of Management and recently he received his master of business administration (MBA) degree from Asian Institute of Management, Makati City, Philippines. He had been a manager of Think \& Learn Company before joining in the institute.

Song-Kyoo Kim is a faculty member of Asian Institute of Management, who is working as an associate professor. He had been a technical manager and TRIZ specialist of mobile communication division at Samsung Electronics and he is involved in IT industries more than 10 years. Dr Kim has received his master degree of computer engineering on 1999 and $\mathrm{Ph} . \mathrm{D}$. of operations research on 2002 from Florida Institute of Technology. He is the author of more than 20 operations research papers focused on stochastic modeling, systematic innovations and patents. He had been the project leader of several 6 Sigma and TRIZ projects mainly focused on the mobile industry. 